Kidney Blood Press Res 1996;19:401-402

\title{
Subject Index Vol. 19, 1996
}

Ablation nephropathy 241

Acute renal failure 38

Adenosine analogues 100

Adenosine-binding protein 100

S-Adenosylhomocysteine hydrolase 100

Adhesion 166

Adrenals 109

Advanced glucosylation end products 305

Amiloride 24

1 >・Aminohippurate transport 332

Analogue, vitamin D 325

Angiotensin, generation 254

Anion channels 317

Aquaporin 32

Arginine vasopressin 81

L-Arginine 177

AT2 receptors 241

Atomic force microscopy 142

ATP-sensitive $\mathrm{K}+$ channels 142

Autoimmunity 298

Basolateral membrane 230

Bicarbonate 87

Bio-impedance 94

Blood pressure 340

Body water 94

Brush border membrane 151, 230

Ca2+influx 148

signaling 148 Calcineurin 174 Calcium 81, 172

antagonist cAMP 81 Catecholamines 109

Cell proliferation and differentiation 325 Chemokines 270 Chemotactic factor 270 Cholesterol 230 Chromosomal localization 209 Chronic renal failure 191

- $\quad$ - -, angiotensin 254

Clinical study 196

Collecting duct 32

Complement function, glomerular injury

290 Cortex, renal 128 Cytokines 177,281

Diabetes 166

- $\quad$ nephropathy 305

Dialysis 94 
Dog kidney 230

L-Dopa 109 Dopamine 109

51Cr-EDTA clearance 121

Electrophysiology 155

Endocytosis 142

Endothelin-1 182

Endothelium 166

Enzyme activity 100

Epithelia 148

Experimental progressive nephropathies

182 Extracellular matrix 184,281, 305

Fatty acids 230

Focal segmental glomerulosclerosis 245

Fura-2 24

Fusion protein 201

G proteins 172 Gene defect 209 Genetics 172 Glomerular architecture 245

disease 270

epithelial cell 81

filtration rate 340

- -, pregnancy 121

immunological injury 290 Glomerulonephritis 177, 196,201,263,

270,281,298 Glomerulosclerosis 177,182,254 Glomerulus 184,254,281 Goodpasture’s syndrome 298 Growth factors 254,281

Hemodialysis 215 Hemodynamics 225 Hemolytic uremic syndrome 205 Hemorrhage 340

Hepatitis C 215 Hereditary nephropathies 209 Heterogeneity 209 Human disease 160

Humanized antibodies 174 Hyperglycemia 305 Hypertension 160, 166, 172, 177 Hypotension

160

Immune complexes, glomerular injury 2 Immunodeficiency 174 Immunosuppression 174

Immunosuppressive therapy 201,298 Inflammation 177

Inner medullary collecting duct 317

Integrins 184

Interferon 215

Interstitial nephritis 298

Inulin clearance 121

Ischemia 332

Kidney 32,109,115,160,184

pathophysiology 225

transplantation 201

Lipid peroxidation 263 Lithium clearance 16 Low-phosphate diet 151

Macrophages 177, 305 MDCK cells 142 Medulla, renal 128 Membrane fluidity 230

- $\quad$ vesicles 332

Mercuric chloride 38

Mesangial cell 184

Mesangium 281

Migration 142

Mouse model 160 
Mutations 155

Mycophenolate mofetil 174

Myo-inositol 317

$\mathrm{Na}+/ \mathrm{Ca} 2+$ exchange $24 \mathrm{Na}+/ \mathrm{H}+$ exchange $87 \mathrm{~L}-\mathrm{Name} 340$ Nephropathy 196 Nephrotoxicity 225

Nitric oxide 177,340

- $\quad$ - atherosclerosis 166

OchratoxinA 225 Organ transplantation 174 Organic osmolytes 317 Ouabain 24 Oxytocin 81

Papilla, renal 128 Parathyroid hormone 151 Pathogenesis 191 PD123319 241 Permeability 166

Phorbol esters 87 Phosphate reabsorption 151 Phospholipids 128,230 Podocyte damage 245

Polymerase chain reaction 215

401

Pressure escape 16 Protein kinase C 87, 305

reabsorption 182 Proteinuria 263 Proximal tubular cells 325

tubule 87, 151,225 Pseudoaldosteronism 160

Rabbit kidney 332 Randomized controlled trials 196 Reactive oxygen species 263 Reduced renal perfusion pressure 16 Renal cortex 38

cortical slices 332

function 340

medulla 38

papilla 38

vascular resistance 340 Renin-angiotensin 115

Saline infusion 121

SBFi 24

Scarring 184

Scavenger substances 263

Shock 340

Signal transduction 148,172

Sodium 109

excretion 16, 115

reabsorption 160 Sodium-coupled glucose transporter 155

phosphate transporter 155

sulfate transporter 155 Sodium-independent transporter of neutral

and dibasic amino acids 155 Stilbene derivatives 317 Streptozotocin-induced diabetes 128

Systemic vascular resistance 340

T cells 298

Target of rapamycin 174

Taurine 317

Tetraethylammonium transport 332 Therapy 196 Thrombotic microangiopathy 205

- $\quad$ thrombocytopenic purpura 205

Thyroxine 38

Time course 16

Tissue injury 177

Transforming growth factor beta 305

Transgenic mice 160

Transport molecules 155

Tubular cells 182 
Vasopressin 32

antagonist 32

V2 receptor 32 Viral hepatitis 215 Vitamin D 325

X-linked hypophosphatemia 151

402

Subject Index Vol. 19, 1996 